\title{
Identification of miR-21-5p/TET1-negative regulation pair in the aggressiveness of glioma cells
}

\author{
Zhuo Zhang ${ }^{1}$, Shu-Zhen Yang ${ }^{2}$, You-Fu $\mathbf{Q i}^{3}$, Yong Yin ${ }^{4}$ \\ ${ }^{1}$ Neurology Department, Liaocheng Second People's Hospital, Liaocheng, Shandong, China, ${ }^{2}$ The Second People's Hospital \\ of Shenxian County, Shenxian, Shandong, China, ${ }^{3}$ Department of Neurology, Ward 2, Linqing People's Hospital, Linqing, Shandong, \\ China, ${ }^{4}$ Department of Neurology, Ward 4, People's Hospital of Pingyi County, Pingyi, Shandong, China
}

\begin{abstract}
Increasing evidence highlights that microRNAs (miRNAs) drive glioma initiation and development. Nevertheless, the underlying role of miR-21-5p in glioma is elusive. Hence, we evaluated the underlying role of miR-21-5p in glioma progression. Microarray data analysis provided data indicating that the miR-21-5p level was elevated in glioma. Silenced miR-21-5p suppressed glioma cell growth and invasion. Additionally, our results disclosed that ten-eleven translocation 1 (TET1) was directly targeted by miR-21-5p. Furthermore, antagomir-21-5p restrained glioma cell growth in a xenograft tumour model. In rescue experiments, knockdown of TET1 neutralized miR-21-5p silence-mediated inhibitory function on glioma cell aggressiveness. Taken together, miR-21-5p exerted its carcinogenic effect in glioma cell growth and invasin by targeting TET1.
\end{abstract}

Key words: glioma, proliferation, invasion, MiR-21-5p, TET1.

\section{Introduction}

Glioma is an aggressive brain cancer, characterized by aggressive proliferation, invasion, and dismal prognosis $[3,8]$. Although great advances have been made in therapeutic strategy, the clinical prognosis of patients with glioma remains dismal. Numerous studies have revealed that several abnormal genes are closely related to the progression of glioma. Dysregulated microRNA (miRNAs) has been extensively reported in human disease and particularly in cancer. MiRNAs regulate the expression of mRNAs via binding with their $3^{\prime}-$ UTR [2]. Several miRNAs have been identified as playing a fundamental role in cancer progression, including cellular proliferation, invasion, and metastasis [1].
Recently, upregulation of miR-21-5p has been found in lung cancer, and ectopic expression of miR21-5p promotes cancer cell proliferation [11]. What is more, introduction of miR-21-5p heightens colon cancer cell growth and invasiveness by targeting cell adhesion molecule L1 like (CHL1) [20]. A previous study reported that the expression of miR-21-5p was evidently raised in glioblastoma samples compared to reference normal brain tissues [22]. Although the significance of miR-21-5p in cancer progression has attracted a considerable amount of attention, the pathological correlation and functional importance of miR-21-5p in glioma have not been exploited.

Here, we sought to probe the essential action of the miR-21-5p/TET1 axis in regulating the aggressive 
traits of glioma cells. We established that miR-21$5 p$ was abnormally elevated in human glioma and miR-21-5p silencing attenuated glioma cell growth and invasion. Eventually, we corroborated that miR21-5p exerted its carcinogenic effect in glioma cell growth and invasin by targeting TET1.

\section{Material and methods}

\section{Cell lines and tissues}

Glioma cell lines (U251, T98G, U87MG, and TJ905), and human normal glial cell lines HEB and HEK-293T were obtained from KeyGen Biotech Co. Ltd. (Nanjing, China). Cells were cultured in DMEM (Thermo Fisher Scientific) supplement with 10\% fetal bovine serum (FBS) and 1\% penicillin/streptomycin. Glioma tissues (32 cases) and normal brain specimens (11 cases) were obtained from Liaocheng Second People's Hospital. Normal brain samples were obtained from patients who received brain tissue resection owing to craniocerebral injury. Tissues were stored in liquid nitrogen immediately after surgery. Informed-written consent was signed by involved participants, and this research protocol was approved by the Ethics Committee from Liaocheng Second People's Hospital. The clinicopathological features of all patients are summarized in Table I.

Table I. The clinicopathological features in patients with glioma

\begin{tabular}{|c|c|}
\hline Clinical parameter & Case $(n)$ \\
\hline \multicolumn{2}{|l|}{ Age (years) } \\
\hline$\leq 55$ & 26 \\
\hline$>55$ & 6 \\
\hline \multicolumn{2}{|l|}{ Gender } \\
\hline Female & 19 \\
\hline Male & 13 \\
\hline \multicolumn{2}{|l|}{ Tumour size $(\mathrm{cm})$} \\
\hline$\geq 5$ & 15 \\
\hline$<5$ & 17 \\
\hline \multicolumn{2}{|l|}{ WHO grade } \\
\hline$|\sim| \mid$ & 13 \\
\hline III IV & 19 \\
\hline \multicolumn{2}{|l|}{ KPS score } \\
\hline$\geq 90$ & 8 \\
\hline$<90$ & 24 \\
\hline
\end{tabular}

\section{Cell transfection}

MiR-21-5p inhibitor (anti-miR-21-5p), miR-21-5p mimic, and related negative control (miR-NC, antimiR-NC) were purchased from Sangon Biotech (Shanghai, China). Negative control shRNA (sh-NC) and shRNA targeting TET1 (sh-TET1) were obtained from GenePharma (Shanghai, China). Cell transfections were conducted by use of a Lipofectamine 3000 kit (Thermo Fisher Scientific). Sequences were as follows: miRNA-21-5p, 5'-UAGCUUAUCAGACUGAUGUUGA-3' and miR-21-5p inhibitor (antimiR-21-5p), 5'-TCAACATCAGTCTGATAAGCTA-3'.

\section{Cell proliferation assay}

U251 or T98G cells $\left(2 \times 10^{5}\right.$ cells $\left./ \mathrm{ml}\right)$ were plated in 96-well plates and incubated overnight. $10 \mu \mathrm{l}$ of Cell Counting Kit-8 (CCK-8, Dojindo, Kumamoto, Japan) solution was added into 96-well plates at $24,48,72$, and $96 \mathrm{~h}$, respectively. After incubating for $2 \mathrm{~h}$ at $37^{\circ} \mathrm{C}$, the optical density (OD) value at each time point was measured using a microplate reader (BioTek, Winooski, VT, USA) at $450 \mathrm{~nm}$. The 5-ethynyl-2'-deoxyuridine (EdU) incorporation assay was performed using an Edu kit (Ribobio, Guangzhou, China) according to the manufacturer's manual. EdU (2 nM/well) was diluted in complete culture medium, and U251 or T98G $\left(1 \times 10^{5}\right)$ were incubated with the dilution for $2 \mathrm{~h}$ at $37^{\circ} \mathrm{C}$. Subsequently, the cells were fixed with $4 \%$ paraformaldehyde for $15 \mathrm{~min}$ at $37^{\circ} \mathrm{C}$, and DAPI was used to counterstain the nuclei for $15 \mathrm{~min}$ at room temperature. The images were acquired using a TE2000 Nikon fluorescence microscope (Nikon Corporation, Tokyo, Japan).

\section{Invasion analysis}

The invasion of cells was studied utilizing a transwell chamber (Millipore) with Matrigel (Corning Inc., USA). $100 \mu$ l of glioma cells $\left(2 \times 10^{5}\right)$ was placed into the top compartment while $500 \mu$ medium supplement with $20 \%$ FBS was added to the lower compartment. After $48 \mathrm{~h}$, the cells in the upper chamber were gently removed with a cotton swab. The cells on the underside of the membrane were fixed using $4 \%$ formaldehyde and stained with $1 \%$ crystal violet. Five fields in each group were randomly selected to count the number of invaded cells under an inverted microscope. 


\section{Colony formation}

Cells $\left(1 \times 10^{3}\right)$ were suspended in medium and plated into 6-well culture plates. Cells were incubated for 2 weeks, and colonies were stained with $0.1 \%$ crystal violet solution.

\section{Luciferase reporter gene assay}

The target genes for miR-21-5p were predicted using miRanda, TargetScan, miRDB, and RNA22. The wild type 3'-UTR sequence of TET1 was inserted into pGL3 luciferase reporter (Promega), referred to as TET1-wt. The mutate type of the 3'-UTR was constructed and inserted into pGL3 plasmid, referred to as TET1-mut. pGL3 plasmid and miR-21-5p were cotransfected into HEK-293T cells [12]. Forty-eight hours post-transfection, the luciferase activity was detected using a Luciferase Reporter Assay System Kit (Promega).

\section{RNA isolation and RT-qPCR}

RNA was prepared utilizing TRIzol reagent kit (Thermo Fisher Scientific). cDNA synthesis was completed using a cDNA synthesis kit (Takara Bio Inc., Shiga, Japan), and a All-in-One ${ }^{\mathrm{TM}}$ miRNA First-Strand cDNA Synthesis Kit (GeneCopoeia, Rockville, MD, USA) was used for miRNA cDNA synthesis. The RT-qPCR was executed using a miRNA qPCR Detection kit (GeneCopoeia) or SYBR ${ }^{\circledR}$ Premix Ex Taq ${ }^{\text {TM }}$ ॥ kit (Takara Bio) on a CFX96 system (Bio-Rad). The PCR primers were as follows: miR-21-5p, 5'-GACTGATGTTGATGTCGGGT-3' (forward) and 5'-GTCAGACAGCCCATCGACT-3' (reverse); TET1, 5'-CATCAGTCAAGACTTTAAGCCCT-3' (forward) and 5'-CGGGTGGTTTAGGTTCTGTTT-3' (reverse); U6, 5'-CTCGCTTCGGCAGCACA-3' (forward) and 5'-AACGCTTCACGAATTTGCGT-3' (reverse); GAPDH, 5'-GGAGCGAGATCCCTCCAAAAT-3' (forward) and 5'-GGCTGTTGTCATACTTCTCATGG-3' (reverse). U6 was regarded as a control for miR-21-5p, and GAPDH was the internal reference for TET1. The relative expressions of genes were measured with the $2^{-\Delta \Delta \mathrm{Ct}}$ method.

\section{Immunoblotting}

Total protein extracted from cells was isolated using 10\% SDS-PAGE and was then transferred onto PVDF membrane. After blocking, the PVDF membrane was incubated with TET1 or GAPDH primary antibody (1 : 1000, Abcam, Cambridge, UK) overnight. Next, the PVDF membrane was immuno- blotted with horseradish peroxidase (HRP)-linked secondary antibody for $2 \mathrm{~h}$. Finally, the blots were determined using an ECL kit (Millipore).

\section{In vivo tumourigenicity}

BALB/c nude mice $(n=12)$ were purchased from Shanghai Slake Laboratory Animal Co., Ltd (Shanghai, China). $100 \mu$ l of T98G cells $\left(2 \times 10^{6}\right)$ was subcutaneously inoculated into nude mice. The tumour sizes were recorded with vernier callipers, and the volume was calculated using the following formula: Tumour volume $=0.5 \times$ length $\times$ width $^{2}$. When the average tumour volume reached approximately $100-150 \mathrm{~mm}^{3}$, the mice were divided into 2 groups (antagomir-NC group, $n=6$; antagomir-21-5p group, $n=6$ ). miRNA antagomirs (antagomirs) are synthetic antagonists of miRNAs as silencing agents of miRNAs [19]. $20 \mathrm{nmol}$ antagomir-21-5p or antagomir-NC was injected intratumorally every 3 days. After 4 weeks, nude mice were euthanized, and the xenograft tumour was excised. Tumour tissues were collected and subjected to immunohistochemical staining using TET1 antibody (1 : 200, Abcam). This study was approved by the Ethics Committee (No. 20191009) of Liaocheng Second People's Hospital.

\section{Statistical analysis}

Statistical analyses were performed using GraphPad Prism 7.0, and all data are shown as mean \pm SD. The statistic difference was calculated using Student's t-test or one-way analysis of variance (ANOVA) followed by Tukey's post hoc test. Correlation between TET1 and miR-21-5p was assessed by Pearson analysis. $P<0.05$ was considered as statistical significance.

\section{Results}

\section{miR-21-5p is upregulated in glioma}

In total 140 dysregulated miRNAs were screened out using the GSE90603 dataset (|Log fold change (FC) $\mid>2$ and $p<0.05$, Fig. 1A) [7]. GSE90603 contained 16 patients with glioblastoma (cancer) and 7 healthy individuals (normal) [7]. miR-21-5p was most highly expressed in glioma patients when compared to normal individuals (Fig. 1B). Next, RT-qPCR was utilized to measure the level of miR-21-5p in tissue samples from the collected cohorts of patients with glioma $(n=32)$. When compared to normal $(n=11)$, glioma exhibited higher expression level of miR-215- 

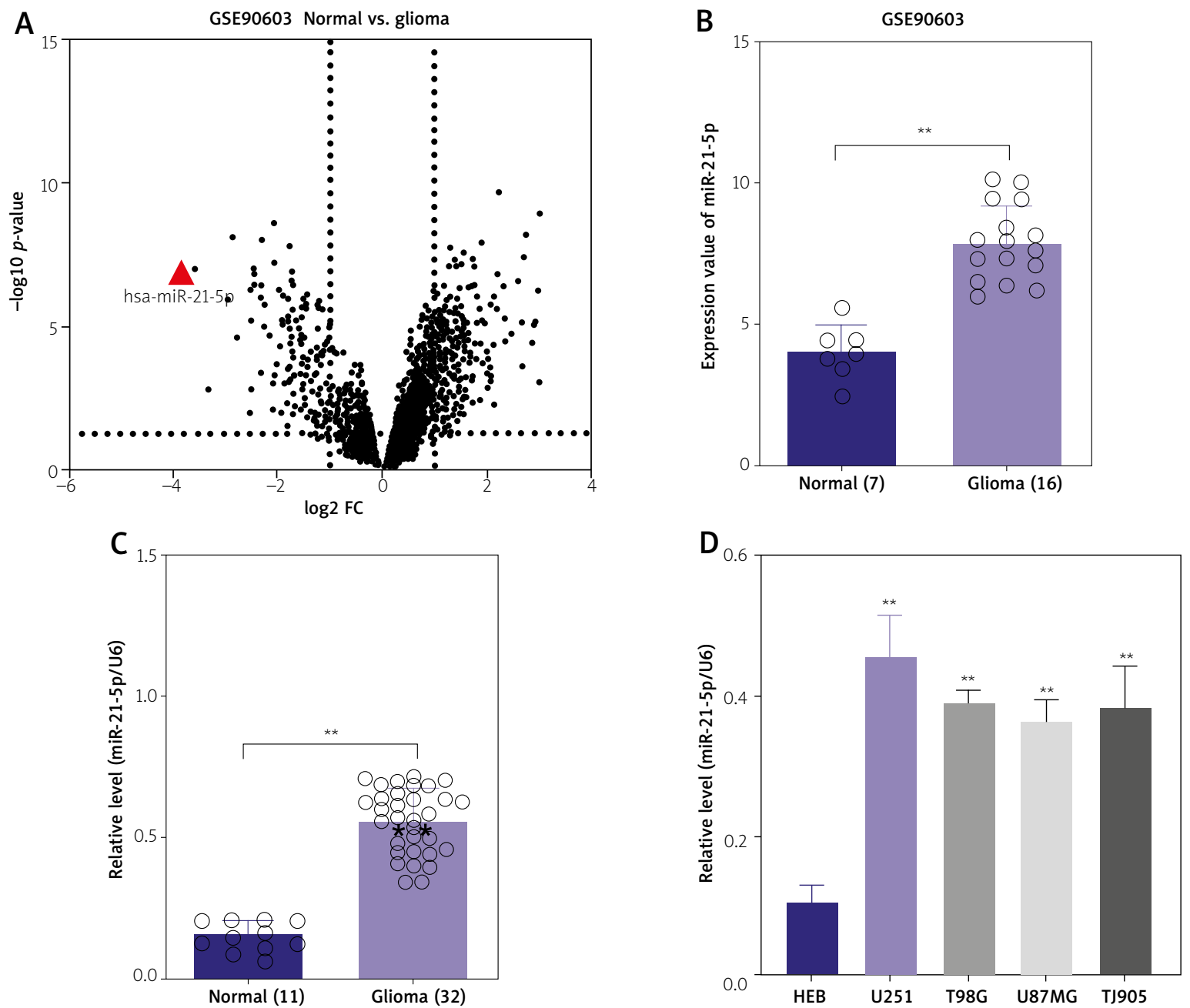

Fig. 1. miR-21-5p is upregulated in glioma tissues and cells. A) Volcano plot showing the significance of the differential miRNA expression in the GSE90603 dataset. The red triangle represents the miR-21-5p. B) According to GSE90603 profiling data, miR-21-5p exhibits a higher expression in glioma. C) Relative expression levels of miR-21-5p in normal and glioma tissues were quantified by RT-qPCR. ${ }^{* *} p<0.01$. D) The expression level of miR-21-5p in glioma cells and normal cell line; HEB was measured by RT-qPCR. ${ }^{* *} p<0.01$ compared to HEB.

5p (Fig. 1C). Compared with the normal HEB cell line, miR-21-5p was extremely over-expressed in glioma cells (U251, T98G, U87MG, and TJ905) (Fig. 1D). These observations collectively show that in glioma, the miR-21-5p level is upregulated.

\section{Glioma cell proliferation is inhibited by knockdown of miR-21-5p}

MiR-21-5p inhibitor (anti-miR-21-5p) was transfected into U251 and T98G cells to decrease the endogenous miR-21-5p level. The results for RT-qPCR revealed that anti-miR-21-5p caused a drastic downregulation of miR-21-5p (Fig. 2A). The role of miR21-5 $p$ in glioma cell proliferation was subsequently elaborated. Compared with the anti-miR-NC group, anti-miR-21-5p reduced $U 251$ and T98G cell proliferation, as demonstrated by CCK-8 ( $p<0.01$, Fig. 2B, C). Glioma cell proliferation was further determined by EdU assay (Fig. 2D). As for transwell invasion assay, anti-miR-21-5p dramatically repressed U251 and T98G cell invasion (Fig. 2E). The aforementioned findings revealed that silence of miR-21-5p impaired glioma cell proliferation and its invasiveness. 

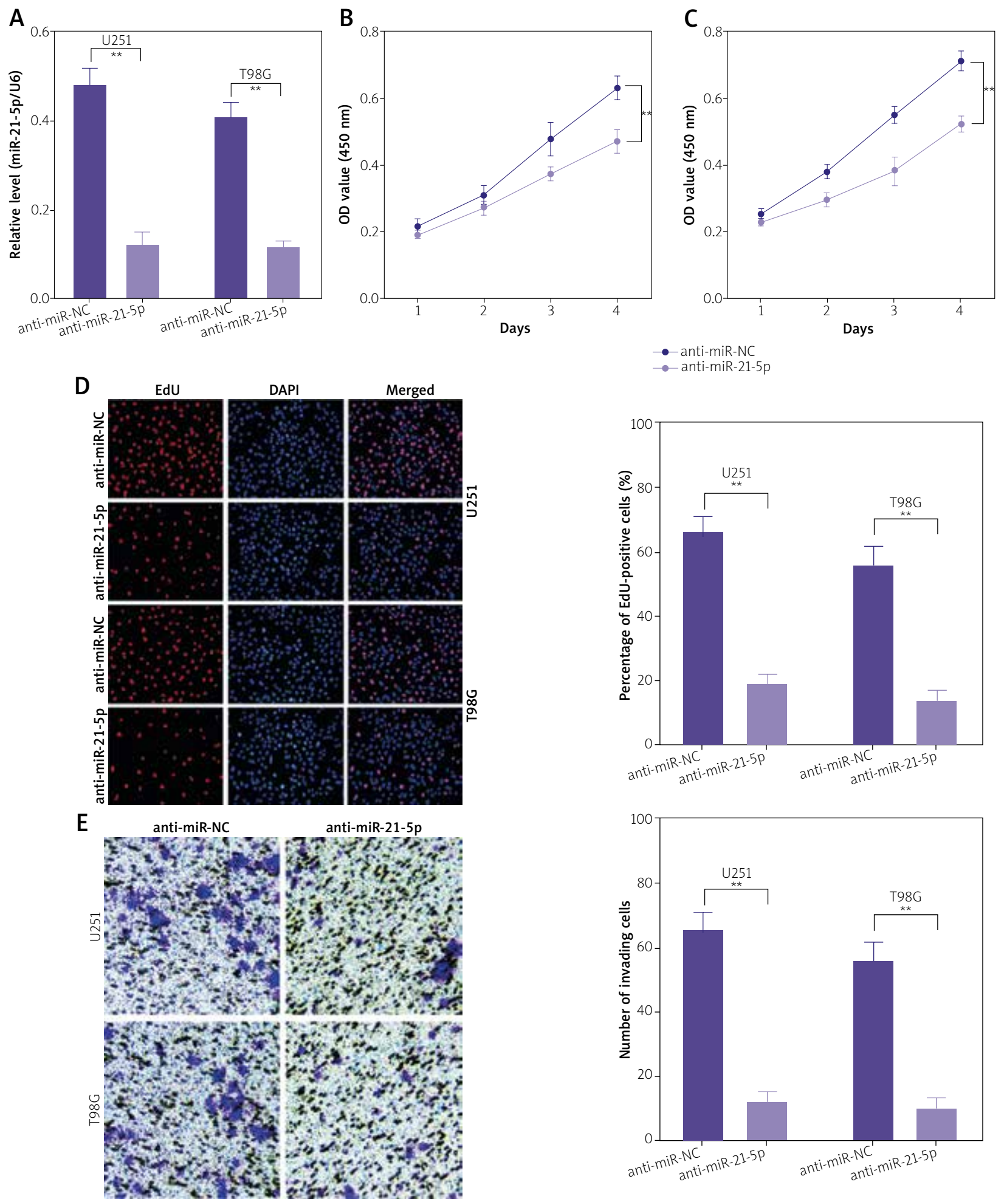

Fig. 2. Glioma cell proliferation and invasion is repressed by miR-21-5p inhibitor. A) U251 and T98G cells were transfected with anti-miR-NC or anti-miR-21-5p, respectively. The level of miR-21-5p was determined by RT-qPCR. B, C) Effects of anti-miR-21-5p on U251 and T98G cell proliferation were measured by CCK-8 assay. D) Cell proliferation assessment after inhibition of miR-21-5p evaluated by EdU assay. E) Representative images and bar graphs depicting the invasion abilities of miR-21-5p-silenced U251 and T98G. ${ }^{* *} p<0.01$. 


\section{Knockdown of miR-21-5p represses T98G cell growth in vivo}

To elucidate the influence of miR-21-5p on glioma cell growth, a colony-forming assay was used. As presented in Figure 3A, B, anti-miR-21-5p weakened the colony-forming ability of U251 and T98G cells ( $p<0.01$ ). In a T98G cell transplantation model, tumour growth was weakened in mice injected with antagomir-21-5p (Fig. 3C-E). These data collectively implied that miR-21-5p inhibitor prevented the tumour growth of T98G cells in vivo.

\section{TET1 is directly targeted by miR-21-5p}

To determine the genes targeted by miR-21-5p, bioinformatics analysis was subsequently performed using miRanda, TargetScan, miRDB, and RNA22. TET1 3'-UTR contains a putative miR-21-5p binding site (Fig. 4A, B). In luciferase reporter gene test, miR21-5p mimic lowered luciferase activity of reporter plasmid carrying wt TET1 3'-UTR but not mut TET1 3'-UTR (Fig. 4C). When compared to normal tissue, glioma tissues exhibited lower expression levels of TET1 (Fig. 4D). Pearson's correlation of miR-21-

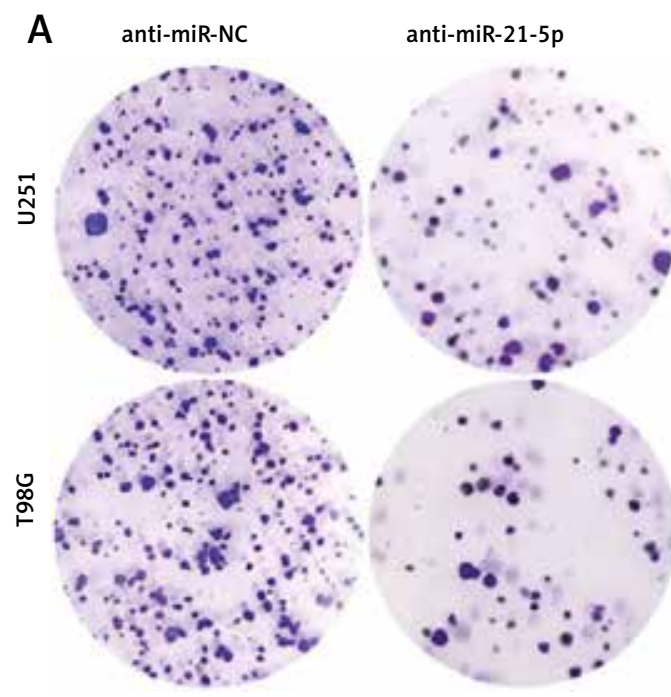

B
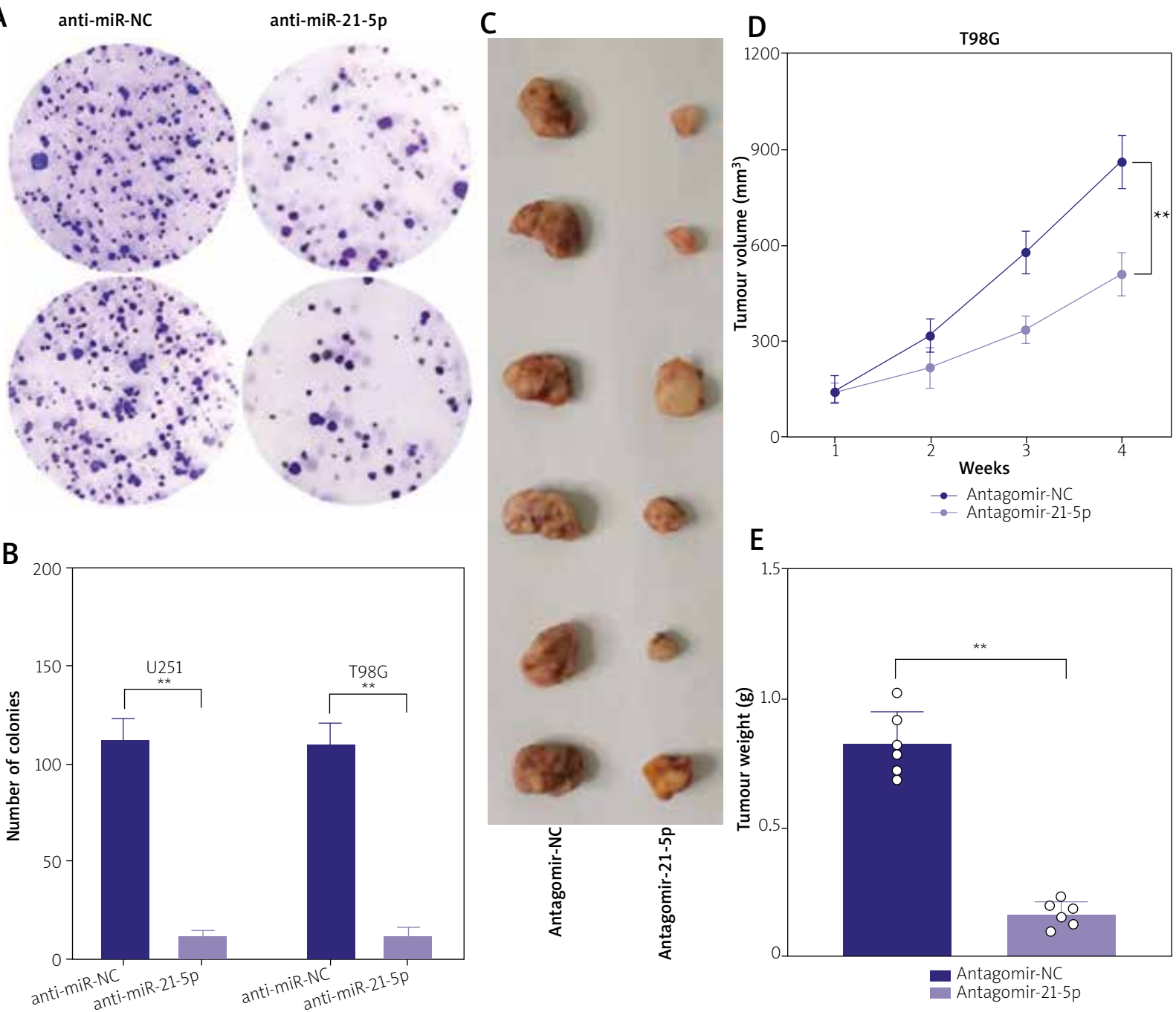

E

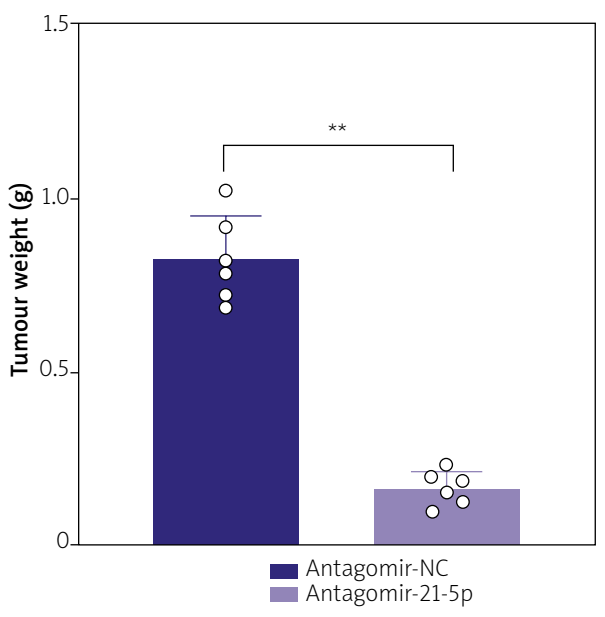

Fig. 3. miR-21-5p inhibitor inhibits glioma cell growth in vivo. A, B) Effects of anti-miR-21-5p on colony formation in U251 and T98G cell. C) Subcutaneous tumours formed from the injection of T98G cells into nude mice. D) Tumour growth curve. E) Tumour weight. ${ }^{* *} p<0.01$. 

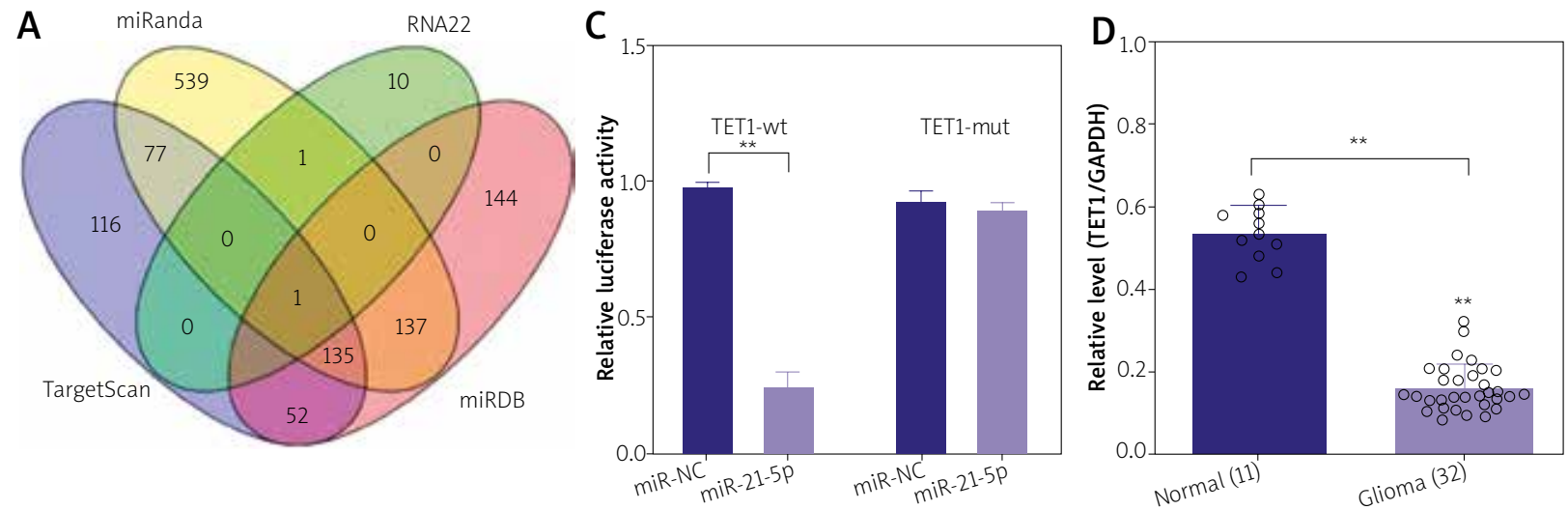

B

wt TET1 3'-UTR 5' ...UCCACACAGUUAAAAAUAAUCUG...

miR-21-5p 3' AGUUGUAGUCAGAC--UAUUCGAU

MUT TET1 3'-UTR 5 ...UCCACACAGUUAAAAUAUUCUAG...
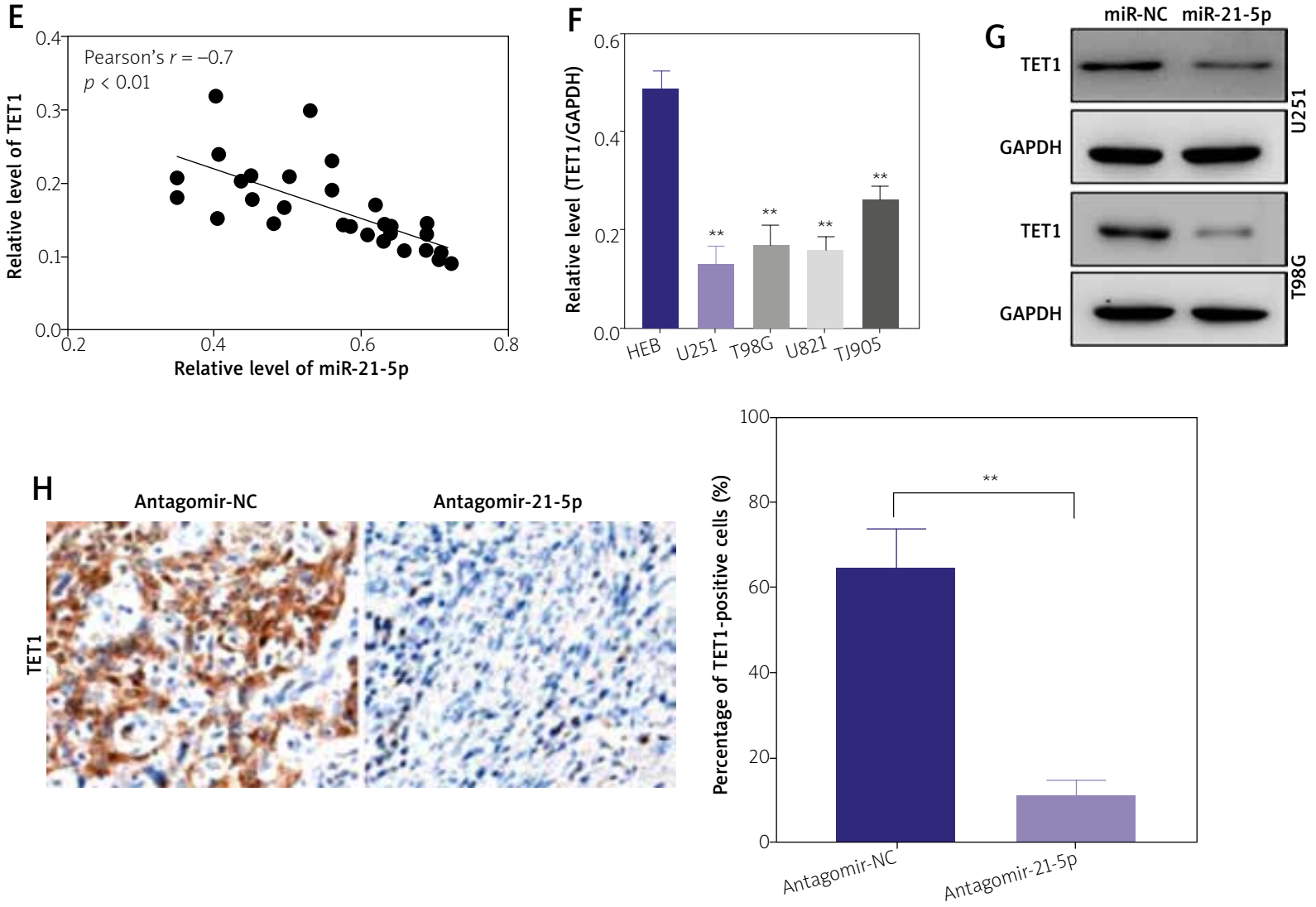

Fig. 4. TET1 is negatively regulated by miR-21-5p. A) Target gene prediction was performed using the online software miRanda, TargetScan, miRDB, and RNA22. Venn diagram showing the common target from 4 bioinformatics tools. B) Prediction of binding sites between 3'-UTR of TET1 and miR-21-5p. C) The binding relationship of TET1 with miR-21-5p confirmed by luciferase reporter gene assay. D) Relative expression levels of TET1 in normal and glioma tissues were quantified by RT-qPCR. E) Pearson's correlation analysis was performed to analyse the correlation between miR-21-5p and TET1 in glioma tissues. F) The expression level of TET1 in glioma cells and normal cell line HEB was measured by RT-qPCR. ${ }^{* *} p<0.01$ compared to HEB. G) U251 and T98G cells were transfected with miR-NC or miR-21-5p mimic. The protein expression of TET1 was detected using western blot. H) Immunohistochemical staining for TET1 in tumour tissue sections from mice injected with antagomir-21-5p or antagomir-21-5p (left panel). Quantitative analysis of TET1-positive staining of cells (right panel). ${ }^{* *} p<0.01$. 
$5 p$ and TET1 was calculated using glioma tissues, and we observed that the TET1 level was negatively correlated with the miR-21-5p level (Fig. 4E). We then determined the mRNA level of TET1 and revealed that it was lowly expressed in U251 and T98G cells compared to HEB cells (Fig. 4F). Moreover, TET1 expressions in both U251 and T98G cells were reduced by miR-21-5p (Fig. 4G). The results of immunohistochemical staining showed that tumour tissue derived from mice treated with antagomir-21-5p exhibited lower expression of TET1 (Fig. 4H). Altogether, these data affirmed that miR-21-5p directly recognizes the 3'-UTR of TET1.

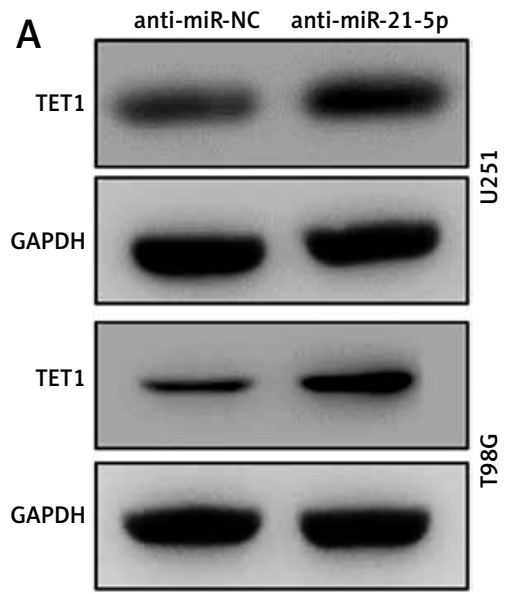

C

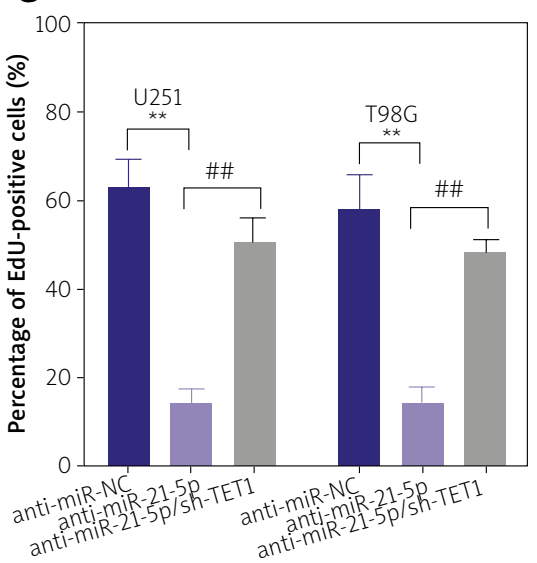

D

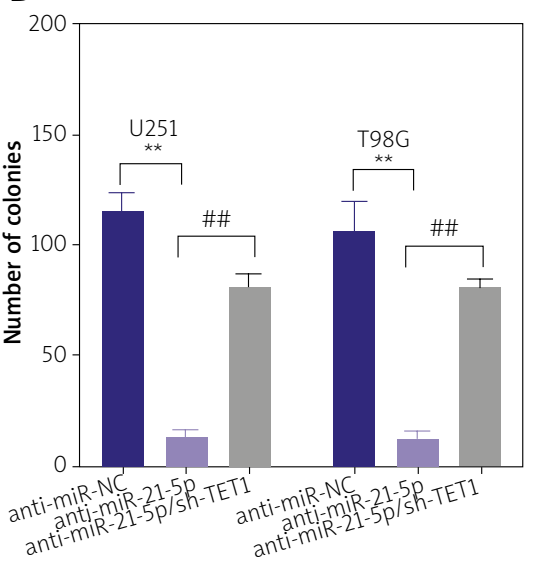

E

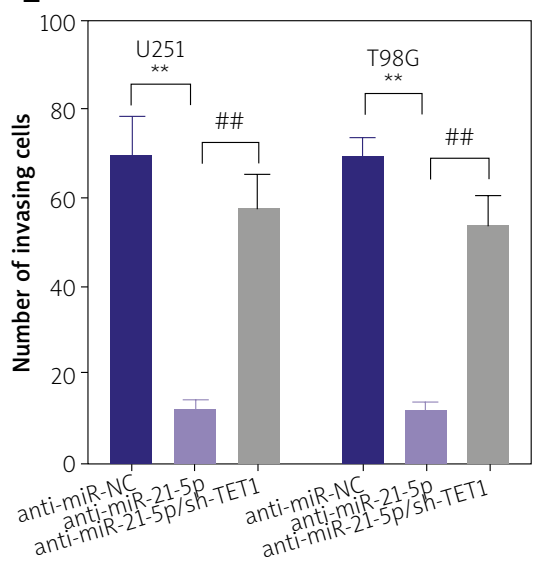

Fig. 5. TET1 mediates the tumour-inhibiting functions of anti-miR-21-5p in glioma cells. A) U251 and T98G cell was transfected with anti-miR-NC or anti-miR-21-5p. The protein expression of TET1 was detected using western blot. B) shRNA TET1 (sh-TET1) and anti-miR-21-5p were co-transfected into U251 and T98G cells. The expression of TET1 was assessed using immunoblotting. C, D) Decreased cell growth in antimiR-21-5p transfected U251 and T98G cells was abolished by TET1 knockdown. E) Decreased invasion in anti-miR-21-5p transfected U251 and T98G cells was abolished by TET1 knockdown. ${ }^{* *} p<0.01,{ }^{\# \#} p<0.01$. 
decreased TET1 expression, which was boosted upon anti-miR-21-5p transfection (Fig. 5B). Intriguingly, anti-miR-21-5p remarkably restrained glioma cell growth and invasion, respectively (Fig. 5C-E). The co-transfection group reversed the decrease in U251 and T98G cell proliferation and invasion caused by miR-21-5p downregulation (Fig. 5C-E). These results collectively showed that TET1 silencing offset the inhibition effect of anti-miR-21-5p.

\section{Discussion}

Growing evidence validates that miRNAs exert uniquely crucial roles in the pathogenesis of various cancers, including glioma [14]. Herein, we clarified the function of miR-21-5p in the growth and invasive trait of glioma cells. The key findings demonstrated that miR-21-5p targets TET1, and its descending expression ultimately hinders glioma cell proliferation and invasion.

Recently, upregulation of miR-21-5p has been found in lung adenocarcinoma (LUAD), and miR21-5p facilitates LUAD cell invasion and metastasis through targeting of WW and C2 domain containing 2 (WWC2) [18]. Moreover, miR-21-5p might be a promising biomarker in multiple tumour types, including pancreatic cancer, rectal cancer, and gastric carcinoma $[13,16,17]$. A previous report presented evidence highlighting the upregulation of miR-21-5p in glioma cell lines and tissues [22]. With the aid of bioinformatics analysis, overexpression of miR-21 has been confirmed as a potential independent prognostic biomarker of poorer overall survival for glioma patients [9]. While its biological function in glioma is urgent to be clarified. In line with reported articles, we discovered that miR-21-5p was highly expressed and TET1 was downregulated in glioma tissues. From our results, we concluded that miR-21-5p had a vital influence on U251 and T98G cell proliferation and invasion. Therefore, our results underline that miR-21-5p exerted an oncogenic function in glioma. Similarly, the oncogenic potential of miR-21-5p in glioma cell growth was confirmed using a nude mice transplanted tumour model. Intratumoural injections of antagomir-21-5p apparently reduced the tumour volumes of transplantation tumours formed by glioma T98G cells.

We used 4 miRNA-target prediction algorithms to find the potential target of miR-21-5p. The algorithms simultaneously identified that miR-21-5p contains a potential binding site compatible with TET1 3'-UTR. This finding is consistent with a previous study [10]. The binding between miR-21-5p and TET1 3'-UTR was demonstrated utilizing luciferase reporter gene test. Western blot studies implied that TET1 expression was negatively modulated by miR21-5p, further corroborating the interaction between TET1 3'-UTR and miR-21-5p.

A prior study concluded that miR-21-5p directly targets and negatively regulates TET 1 in colorectal cancer cells [4]. TET1 has potent cancer-inhibitive effects in ovarian cancer cells via activation of Dikkopf 1 (DKK1) and secreted Fzd receptor protein 2 (SFRP2) [5]. The expression of TET1 in glioma tissue is significantly lower than the corresponding non-tumour normal tissues [6]. Available evidence has unveiled that TET1 serves as a critical participator in cancer cell metastasis $[15,21]$. In this study, TET1 knockdown significantly facilitated the aggressive traits of glioma cells, which was impaired by miR-21$5 p$ depletion. These findings suggest that miR-21-5p exerted its carcinogenic effect in glioma cell growth and invasin by targeting TET1. There remain insufficiencies in this research. The role of the miR-21-5p/ TET1 axis should be further explored in rescue assay using a corresponding subcutaneous tumorigenesis experiment. Finally, the clinical sample size was insufficient. Further studies in larger cohorts will assist in exploring the prognostic value of miR-21-5p in glioma.

Our results illustrate the cancer-promoting role miR-21-5p in glioma, and that downregulation of miR-21-5p inhibits glioma cell growth and invasion. Altogether, this study illustrates the potential of miR-21-5p as a therapeutic target in glioma.

\section{Disclosure}

The authors report no conflict of interest.

\section{References}

1. Ali Syeda Z, Langden SSS, Munkhzul C, Lee M, Song SJ. Regulatory mechanism of microRNA expression in cancer. Int J Mol Sci 2020; 21: 1723

2. Babaei K, Shams S, Keymoradzadeh A, Vahidi S, Hamami P, Khaksar R, Norollahi SE, Samadani AA. An insight of microRNAs performance in carcinogenesis and tumorigenesis; an overview of cancer therapy. Life Sci 2020; 240: 117077.

3. Babarczy K, Reisz Z, Szabo E, Rajda C, Vecsei L, Bodi I, Klivenyi P, Hortobagyi T, Szalardy L. A longitudinally extensive $\mathrm{H} 3$ K27M-mutant diffuse midline glioma in an elderly patient clin- 
ically mimicking central nervous system inflammation: a case report. Folia Neuropathol 2020; 58: 377-385.

4. Cheng YW, Chou CJ, Yang PM. Ten-eleven translocation 1 (TET1) gene is a potential target of miR-21-5p in human colorectal cancer. Surg Oncol 2018; 27: 76-81.

5. Duan H, Yan Z, Chen W, Wu Y, Han J, Guo H, Qiao J. TET1 inhibits EMT of ovarian cancer cells through activating Wnt/beta-catenin signaling inhibitors DKK1 and SFRP2. Gynecol Oncol 2017; 147: 408-417.

6. Fu R, Ding Y, Luo J, Yu L, Li CL, Li DS, Guo SW. TET1 exerts its tumour suppressor function by regulating autophagy in glioma cells. Biosci Rep 2017; 37: BSR20160523.

7. Gulluoglu S, Tuysuz EC, Sahin M, Kuskucu A, Kaan Yaltirik C, Ture U, Kucukkaraduman B, Akbar MW, Gure AO, Bayrak OF, Dalan AB. Simultaneous miRNA and mRNA transcriptome profiling of glioblastoma samples reveals a novel set of OncomiR candidates and their target genes. Brain Res 2018; 1700: 199-210.

8. Huang W, Shi Y, Han B, Wang Q, Zhang B, Qi C, Liu F. LncRNA GAS5-AS1 inhibits glioma proliferation, migration, and invasion via miR-106b-5p/TUSC2 axis. Human Cell 2020; 33: 416-426.

9. Jiang G, Mu J, Liu X, Peng X, Zhong F, Yuan W, Deng F, Peng X, Peng S, Zeng $X$. Prognostic value of miR-21 in gliomas: comprehensive study based on meta-analysis and TCGA dataset validation. Sci Rep 2020; 10: 4220.

10. Li GQ, Fang YX, Liu Y, Meng FR, Wu X, Zhang CW, Zhang Y, Liu YQ, Liu D. MicroRNA-21 from bone marrow mesenchymal stem cell-derived extracellular vesicles targets TET1 to suppress KLF4 and alleviate rheumatoid arthritis. Ther Adv Chronic Dis 2021; 12: 20406223211007369.

11. Li X, Wu X. MiR-21-5p promotes the progression of non-smallcell lung cancer by regulating the expression of SMAD7. Onco Targets Ther 2018; 11: 8445-8454.

12. Liang TS, Zheng YJ, Wang J, Zhao JY, Yang DK, Liu ZS. MicroRNA506 inhibits tumor growth and metastasis in nasopharyngeal carcinoma through the inactivation of the Wnt/beta-catenin signaling pathway by down-regulating LHX2. J Exp Clin Cancer Res 2019; 38: 97.

13. Lopes-Ramos CM, Habr-Gama A, Quevedo BdeS, Felicio NM, Bettoni F, Koyama FC, Asprino PF, Galante PA, Gama-Rodrigues J, Camargo AA, Perez RO, Parmigiani RB. Overexpression of miR21-5 $p$ as a predictive marker for complete tumor regression to neoadjuvant chemoradiotherapy in rectal cancer patients. BMC Med Genomics 2014; 7: 68.

14. Lucero R, Zappulli V, Sammarco A, Murillo OD, Cheah PS, Srinivasan S, Tai E, Ting DT, Wei Z, Roth ME, Laurent LC, Krichevsky AM, Breakefield XO, Milosavljevic A. Glioma-derived miRNA-containing extracellular vesicles induce angiogenesis by reprogramming brain endothelial cells. Cell Rep 2020; 30 2065-2074.

15. Ma L, Qi T, Wang S, Hao M, Sakhawat A, Liang T, Zhang L, Cong X, Huang Y. Tet methylcytosine dioxygenase 1 promotes hypoxic gene induction and cell migration in colon cancer. J Cell Physiol 2019; 234: 6286-6297.

16. Park SK, Park YS, Ahn JY, Do EJ, Kim D, Kim JE, Jung K, Byeon JS, Ye BD, Yang DH, Park SH, Hwang SW, Jung HY, Myung SJ. MiR- 21-5p as a predictor of recurrence in young gastric cancer patients. J Gastroenterol Hepatol 2016; 31: 1429-1435.

17. Qu K, Zhang X, Lin T, Liu T, Wang Z, Liu S, Zhou L, Wei J, Chang H, Li K, Wang Z, LiU C, Wu Z. Circulating miRNA-21-5p as a diagnostic biomarker for pancreatic cancer: evidence from comprehensive miRNA expression profiling analysis and clinical validation. Sci Rep 2017; 7: 1692.

18. Wang G, Zhou Y, Chen W, Yang Y, Ye J, Ou H, Wu H. miR-21-5p promotes lung adenocarcinoma cell proliferation, migration and invasion via targeting WWC2. Cancer Biomark 2020; 28 : 549-559.

19. Yang X, Tang X, Sun P, Shi Y, Liu K, Hassan SH, Stetler RA, Chen J, Yin KJ. MicroRNA-15a/16-1 antagomir ameliorates ischemic brain injury in experimental stroke. Stroke 2017; 48: 1941-1947.

20. Yu W, Zhu K, Wang Y, Yu H, Guo J. Overexpression of miR-21-5p promotes proliferation and invasion of colon adenocarcinoma cells through targeting CHL1. Mol Med 2018; 24: 36.

21. Zhou Z, Zhang HS, Liu Y, Zhang ZG, Du GY, Li H, Yu XY, Huang YH. Loss of TET1 facilitates DLD1 colon cancer cell migration via H3K27me3-mediated down-regulation of E-cadherin. J Cell Physiol 2018; 233: 1359-1369.

22. Zottel A, Samec N, Kump A, Raspor Dall'Olio LR, Puzar Dominkus P, Romih R, Hudoklin S, Mlakar J, Nikitin D, Sorokin M, Buzdin A, Jovcevska I, Komel R. Analysis of miR-9-5p, miR-124-3p, miR21-5p, miR-138-5p, and miR-1-3p in glioblastoma cell lines and extracellular vesicles. Int J Mol Sci 2020; 21: 8491. 